\title{
Simulation Analysis of Vertical Interconnection Column Misalignment during LTCC Laminating Process
}

\author{
Tianhong Luo ${ }^{1, a}$, Dejian Zhou ${ }^{1, b}$ \\ ${ }^{1}$ School of Electromechanical Engineering, Guilin University of Electronic Technology, Guilin, \\ 541004, China \\ a460967986@qq.com, bemezdj@guet.edu.cn
}

Keywords: LTCC, laminating process, vertical interconnection column, misalignment, simulation.

\begin{abstract}
A three-dimensional model of LTCC microwave component was built by the finite element software ANSYS, and the influence of LTCC laminating process parameters to vertical interconnection column misalignment was studied by simulation analysis. Results show that the closer to the edge of component, the greater vertical interconnection column misalignment. Pressure is the main factor that leads to the misalignment of vertical interconnection column. Choose laminating process parameters combination by pressure of $10 \mathrm{MPa}$, temperature of $90{ }^{\circ} \mathrm{C}$ and time of $6 \mathrm{~min}$, the vertical interconnection column misalignment is $2.92 \%$, down to below $3 \%$.
\end{abstract}

\section{Introduction}

As the electronic products of application platform such as aviation, aerospace increasingly miniaturization, multi-functional, lightweight, high frequency, and the integration of electronic system put forward higher requirements. LTCC ( Low Temperature Co-Fired Ceramic) with its superior electrical characteristics and advanced production technology has attracted much attention in the field of high density and high frequency applications[1]. In order to adapt to the need of high density interconnect circuit, a variety of fine interconnection technology based on LTCC has developed. The LTCC integrated system which use vertical interconnection column to electrical interconnection, thermal conductivity has superior properties such as high integration, short interconnect line, good high frequency performance, etc. At the same time, using vertical interconnection column to thermal conductivity also effectively solve the problem of system heat dissipation. However, high density interconnect encapsulation put forward higher request to the manufacturing process of LTCC multi-layer board inside vertical interconnection column. If vertical interconnection column generate misalignment defect in production process, the LTCC internal electrical interconnection system would appear problem such as open circuit, short circuit, and greatly reduce the expected effect of heat conduction. In order to reduce the misalignment, In this paper, a three-dimensional model of LTCC microwave component is established by the finite element software ANSYS, and the influence of LTCC laminating process parameters to vertical interconnection column misalignment is studied by simulation analysis. Eventually get LTCC laminating process parameters combination which make smaller misalignment. The results of this paper has a certain theoretical guiding significance to production.

\section{LTCC laminating process and LTCC constitutive model}

LTCC laminating process use warm water isostatic pressing. The water heated to a certain temperature and maintain, then put LTCC green tape in water, make it's every directions receive equivalent pressure and keep a certain amount of time. In this paper, viscoelastic constitutive model will be used for LTCC green tape[2]. In the finite element software ANSYS generalized Prony model is used to describe the viscoelastic properties of material, generalized Prony model can better reflect the viscoelastic properties of material[3]. The basic form of using Prony series to express viscoelastic properties can be given as follow: 


$$
\begin{array}{r}
G(t)=G_{\infty}+\sum_{i=1}^{n_{G}} G_{i} \exp \left(-\frac{t}{\tau_{i}^{G}}\right) \\
K(t)=K_{\infty}+\sum_{i=1}^{n_{K}} K_{i} \exp \left(-\frac{t}{\tau_{i}^{K}}\right)
\end{array}
$$

Where $G_{\infty}$ and $G_{i}$ are shear modulus, $K_{\infty}$ and $K_{i}$ are bulk modulus, $\tau_{i}^{G}$ and $\tau_{i}^{K}$ are the relaxation time of Prony series[4].

\section{Simulation model}

The LTCC microwave component analyzed in this paper as shown in figure 1. It is manufactured by 25 layers of LTCC green tape and thickness is $0.127 \mathrm{~mm}$ per layer, the total thickness is $3.175 \mathrm{~mm}, 50 \mathrm{~mm}$ in length and $40 \mathrm{~mm}$ in width. There are vertical interconnection columns and microchannels within component. The diameter of the vertical interconnection column is $0.18 \mathrm{~mm}$. There are 256 electrically conductive columns and 256 thermally conductive colums. The electrically conductive columns provide electrical interconnection for circuit on each layer of LTCC. The thermally conductive colums connect power chip and branch channel, transfer the heat generated in the chip into branch channel, for cooling function. Because the structure of component is symmetrical, and the loads and boundary conditions are symmetrical too, in order to save the time of simulation calculation, half of the overall model is built for simulation analysis finally.

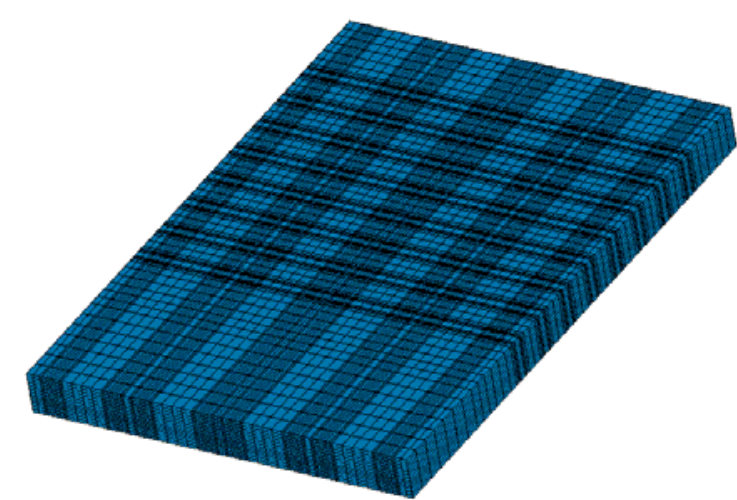

Fig. 1 LTCC microwave component module

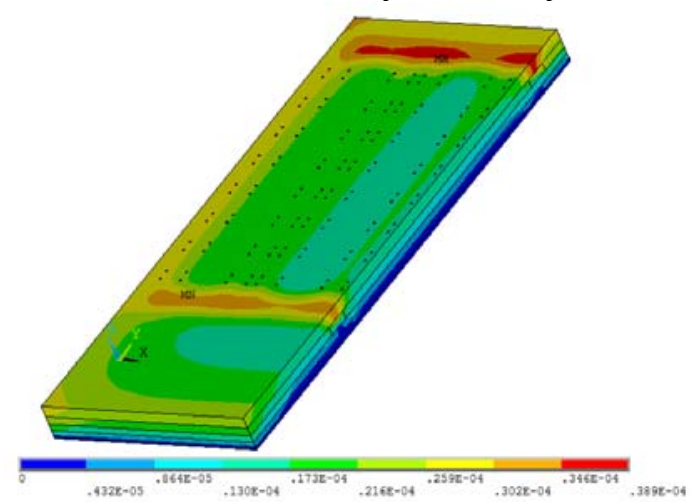

Fig. 2 Total displacement distribution of module

\section{Simulation analysis}

\subsection{Typical LTCC laminating process analysis.}

The main process parameters of LTCC laminating process are pressure, temperature and time. The typical LTCC laminating process parameters universally applicable in industry are $20 \mathrm{MPa}$ at $70{ }^{\circ} \mathrm{C}$ for $10 \mathrm{~min}[5]$. The LTCC microwave component was simulated under typical LTCC laminating process. After simulation, get the total displacement distribution of component, as shown in figure 2, the deformation mainly in the edge. Figure 3 is the total displacement distribution of vertical interconnection columns in component, it can be seen that the closer to the edge of the component the greater vertical interconnection column deformation. Accordingly, the closer to the edge of the component the greater vertical interconnection column misalignment. The enlarged view is the vertical interconnection column with maximum misalignment, and the misalignment is $5.94 \%$. 


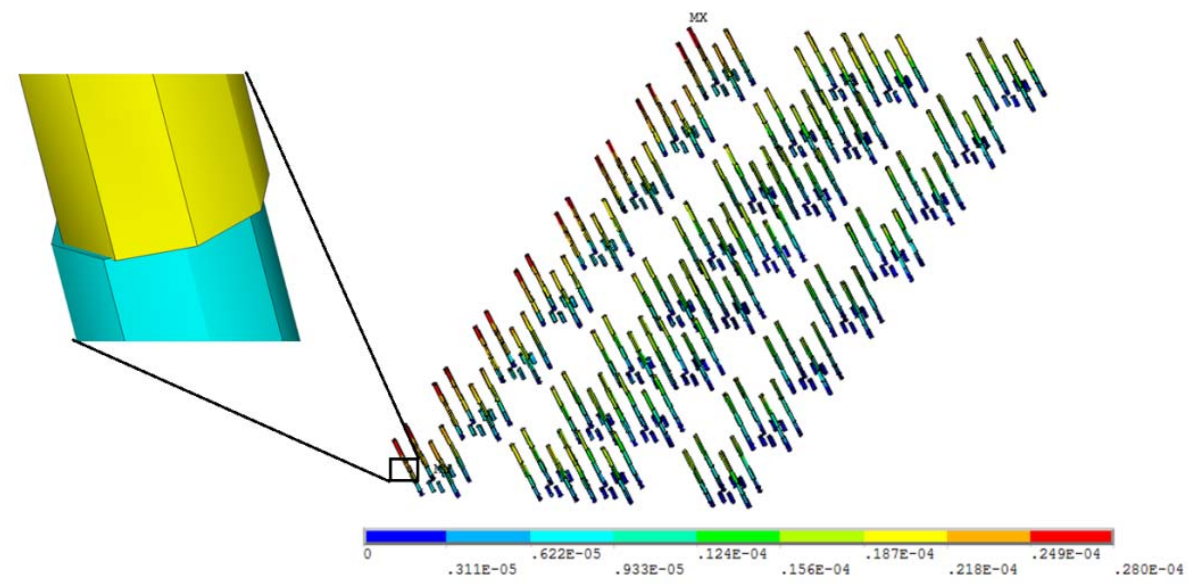

Fig. 2 Total displacement distribution of vertical interconnection columns

4.2 Orthogonal experiment to optimize laminating process.

In practice, LTCC laminating process parameters generally are: pressure takes 10 30MPa, temperature takes $50 \sim 90^{\circ} \mathrm{C}$, time takes $6 \sim 14 \mathrm{Min}$. On the basis of laminating process parameters scope design $L_{9}\left(3^{3}\right)$ orthogonal experiments, the level of three factors as shown in table 1.

Table 1 The orthogonal experiment factors - level

\begin{tabular}{cccc}
\hline Factor & Pressure $(\mathrm{MPa})$ & Temperature $\left({ }^{\circ} \mathrm{C}\right)$ & Time (Min) \\
\hline Level 1 & 10 & 50 & 6 \\
Level 2 & 20 & 70 & 10 \\
Level 3 & 30 & 90 & 14 \\
\hline
\end{tabular}

According to the design of orthogonal experiments, simulation analysis was carried out on the 9 experiments. Respectively to extract each experiment LTCC microwave component inside vertical interconnection column maximum misalignment as the results, and range analysis was carried out on the result data, as shown in table 2.

Table 2 The orthogonal experiment simulation result

\begin{tabular}{ccccc}
\hline Number & Pressure $(\mathrm{MPa})$ & Temperature $\left({ }^{\circ} \mathrm{C}\right)$ & Time $($ Min $)$ & misalignment $(\%)$ \\
\hline 1 & 10 & 50 & 6 & 2.92 \\
2 & 10 & 70 & 10 & 2.99 \\
3 & 10 & 90 & 14 & 3.02 \\
4 & 20 & 50 & 10 & 5.94 \\
5 & 20 & 70 & 14 & 6.01 \\
6 & 20 & 90 & 6 & 5.82 \\
7 & 30 & 50 & 14 & 8.88 \\
8 & 30 & 70 & 6 & 8.60 \\
9 & 30 & 90 & 10 & 8.78 \\
$\mathrm{~K} 1$ & 2.98 & 5.91 & 5.78 & \\
$\mathrm{~K} 2$ & 5.92 & 5.87 & 5.9 & \\
$\mathrm{~K} 3$ & 8.75 & 5.87 & 5.97 & \\
$\mathrm{R}$ & 5.77 & 0.04 & 0.19 & \\
\hline
\end{tabular}

Note: In table $2, \overline{\mathrm{Kn}}$ is the average misalignment of factor on level $\mathrm{n}$; $\mathrm{R}$ is range.

It can be seen from the range analysis in table 2, in these LTCC lamination process parameters, the range value from big to small is: pressure $>$ time $>$ temperature, and the range of pressure is much larger than the range of time and temperature. So in these LTCC lamination process parameters, the influence of pressure on the vertical interconnection column misalignment is the most, and it is far greater than the time and temperature. The diagram of the relationship between LTCC lamination process parameters and vertical interconnection column misalignment, as shown in Figure 4. 


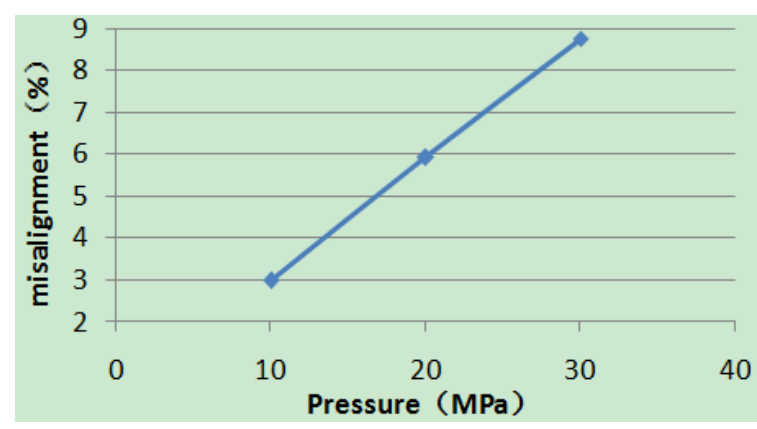

(a) Pressure VS misalignment

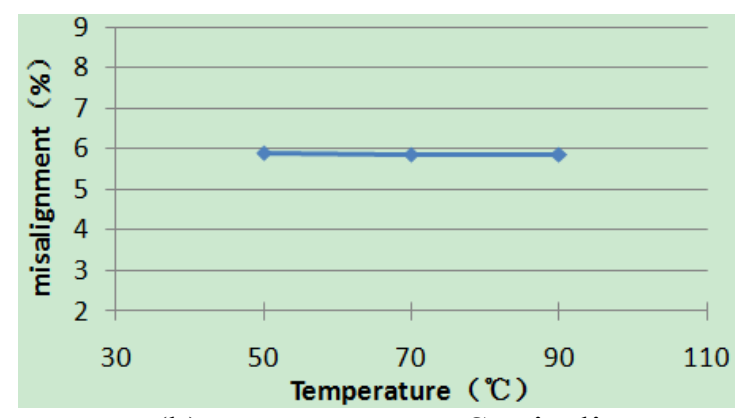

(b) Temperature VS misalignment

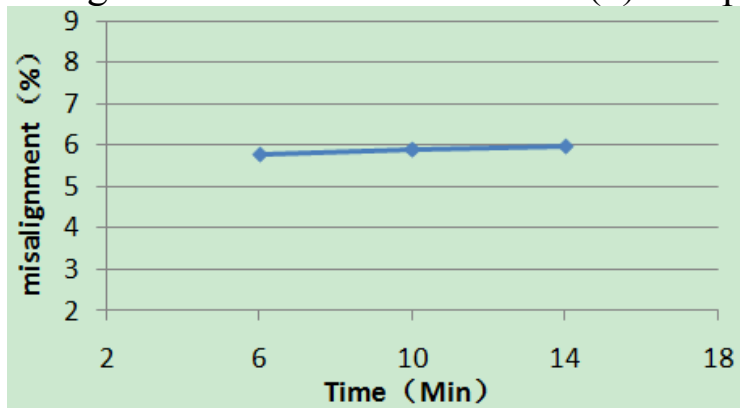

(c) Time VS misalignment

Fig. 4 The relationship between LTCC lamination process parameters and misalignment

From Fig. 4(a), it can be seen that the vertical interconnection column misalignment is approximatively proportional to pressure. When the pressure increased to $30 \mathrm{MPa}$, the average misalignment is $8.75 \%$, it is a little serious. It shows that should reduce the pressure to reduce the misalignment. But the pressure should not less than $10 \mathrm{MPa}$, because if the pressure is too low will lead to delamination. From Fig. 4 (b), it can be seen that the vertical interconnection column misalignment decreases with the increase of the lamination temperature, but the amplitude is small, and when the temperature increased to $70{ }^{\circ} \mathrm{C}$ and continue to increase, the misalignment is almost no longer to reduce. From Fig. 4 (c), it can be seen that the vertical interconnection column misalignment increases with the increase of the lamination time, it shows that can reduce the lamination time appropriate to reduce misalignment.

\section{LTCC laminating process parameters optimization}

Based on the analysis above, through orthogonal experiment analysis to get the optimal combination for laminating process parameters: take $10 \mathrm{MPa}$ pressure, temperature of $90{ }^{\circ} \mathrm{C}$, time of $6 \mathrm{~min}$. According to the optimal laminating process parameters combination, the LTCC microwave component carried out simulation to verify. The result of simulation can be seen in figure 5. After using the optimal LTCC laminating process parameters, the maximum vertical interconnection column misalignment is $2.92 \%$, compared with $5.94 \%$ misalignment under the typical LTCC laminating process parameters combination, decreased by $3.02 \%$, and reduce the misalignment to below $3 \%$. It shows that by using the optimal process parameters combination, the LTCC microwave component can greatly reduces vertical interconnection column misalignment after laminating process. 


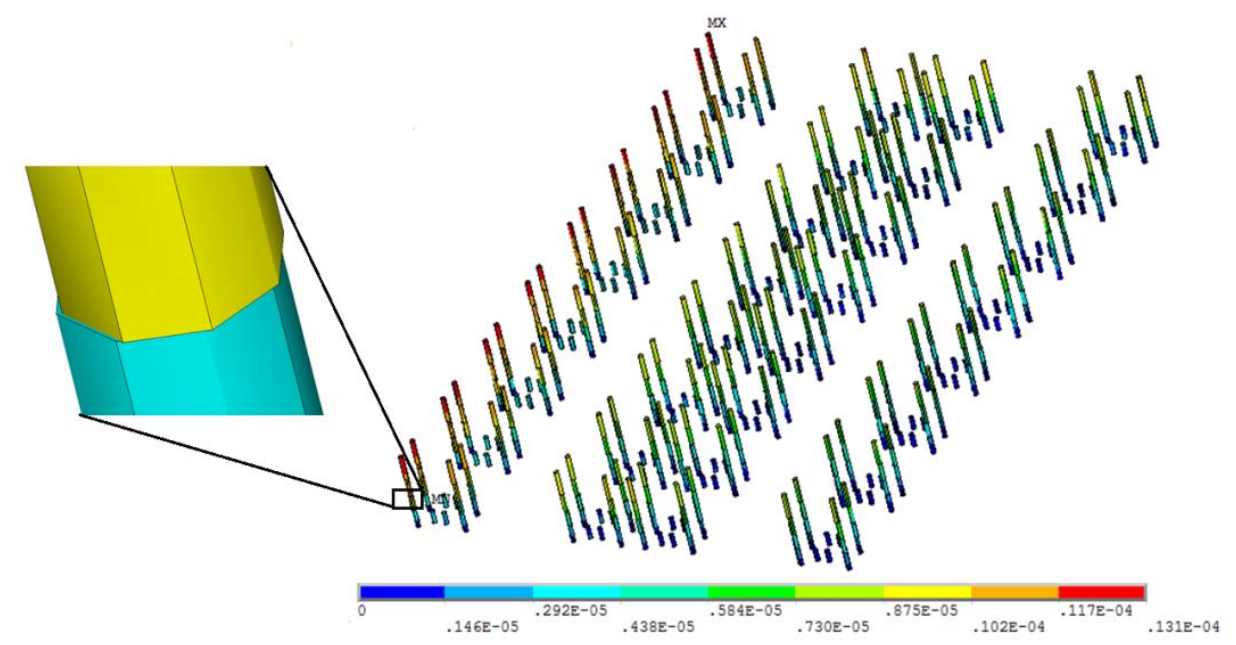

Fig. 5 Total displacement distribution of vertical interconnection columns

\section{Conclusions}

In this paper, a kind of LTCC microwave component inside vertical interconnection column misalignment is studied by ANSYS finite element software after laminating process, and get the following several important conclusions:

(1) The closer to the edge of component, the greater vertical interconnection column misalignment.

(2) Pressure is the main factor that leads to the misalignment of vertical interconnection column, and vertical interconnection column misalignment is approximatively proportional to pressure.

(3) Choose laminating process parameters combination by pressure of $10 \mathrm{MPa}$, temperature of $90{ }^{\circ} \mathrm{C}$ and time of $6 \mathrm{~min}$, the vertical interconnection column misalignment is $2.92 \%$, down to below $3 \%$.

\section{Acknowledgements}

This work was supported by advanced research project "The Study of *** Multi-energy***”.

\section{References}

[1] K. Malecha, L.J. Golonka, Three-dimensional structure of zero-shrinkage LTCC ceramics for micro fluidic applications[J]. Micro-electron Reliable, 2009, 49: 585-591.

[2] Jones WK, Liu Y, Wang P, Zampino M., "Chemical structural and mechanical properties of LTCC tapes” Int J Micro Electron Packaging 2000; 23(4),pp.469-73.

[3] Jianhua Zhang, Lei Ding. ABAQUS introductory and case[M]. Electronic industry press, 2013.

[4] Jiayong Wang. Rutting prediction by FEM and influence factor analysis based on rutting test. [D]. Chang 'an University, 2009.p.18.

[5] Malecha K, Maeder T, Jacq C. Fabrication of membranes and microchannels in low-temperature co-fired ceramic (LTCC) substrate using novel water-based sacrificial carbon pastes[J]. Journal of the European Ceramic Society, 2012,32(12):3277. 